\title{
Liver Transplant Imaging prior to and during the COVID-19 Pandemic
}

\author{
Maria Irene Bellini $\left(\mathbb{D},{ }^{1}\right.$ Daniele Fresilli $\mathbb{D}^{2},{ }^{2}$ Augusto Lauro ${ }^{(D)},{ }^{1}$ Gianluca Mennini $(\mathbb{D})^{3}$ \\ Massimo Rossi $\left(\mathbb{D},{ }^{3}\right.$ Carlo Catalano $\mathbb{D D}^{2},{ }^{2}$ Vito D'Andrea ${ }^{(D)},{ }^{1}$ and Vito Cantisani $(\mathbb{D})^{2}$ \\ ${ }^{1}$ Department of Surgical Sciences, Sapienza University of Rome, Rome, Italy \\ ${ }^{2}$ Department of Radiological, Oncological, Anatomo-Pathological Sciences, Sapienza University of Rome, Rome, Italy \\ ${ }^{3}$ Department of Hepato-Bilopancreatic and Transplant Surgery, Sapienza University of Rome, Rome, Italy
}

Correspondence should be addressed to Maria Irene Bellini; mariairene.bellini@nhs.net

Received 4 June 2021; Accepted 23 December 2021; Published 12 January 2022

Academic Editor: Gernot Kaiser

Copyright (c) 2022 Maria Irene Bellini et al. This is an open access article distributed under the Creative Commons Attribution License, which permits unrestricted use, distribution, and reproduction in any medium, provided the original work is properly cited.

\begin{abstract}
Background. The suspension of the surgical activity, the burden of the infection in immunosuppressed patients, and the comorbidities underlying end-stage organ disease have impacted transplant programs significantly, even life-saving procedures, such as liver transplantation. Methods. A review of the literature was conducted to explore the challenges faced by transplant programs and the adopted strategies to overcome them, with a focus on indications for imaging in liver transplant candidates. Results. Liver transplantation relies on an appropriate imaging method for its success. During the Coronavirus Disease 2019 (COVID-19) pandemic, chest CT showed an additional value to detect early signs of SARS-CoV-2 infection and other screening modalities are less accurate than radiology. Conclusion. There is an emerging recognition of the chest CT value to recommend its use and help COVID-19 detection in patients. This examination appears highly sensitive for liver transplant candidates and recipients, who otherwise would have not undergone it, particularly when asymptomatic.
\end{abstract}

\section{Introduction}

The Coronavirus Disease 2019 (COVID-19) caused by the Severe Acute Respiratory Syndrome Coronavirus-type 2 (SARS-CoV-2) led to unprecedented challenges to healthcare. It is mainly a respiratory disease, with a mild course in most of the cases, but with potentially fatal outcomes for certain population subgroups. Previous reports demonstrated higher risk in immunosuppressed transplant patients, individuals with underlying comorbidities, such as diabetes, age $>70$ years, obesity, and cardiac and/or end-stage organ disease [1]. COVID-19 diagnosis is based on the positivity of the reverse transcriptase-polymerase chain reaction (RT-PCR) [2].

Extreme measures, such as the lockdown or red zone instauration, have been the only effective way to counteract the overwhelming pressure by this novel virus. The aim of this review is to provide a comprehensive assessment of the evolution of the diagnostic imaging caused by the pandemic in relation to liver transplantation (LT). We report the standard of practice running until the prepandemic area, followed by the impact of COVID-19 in the use of radiological means to select, manage, and follow up LT patients and donors during these challenging times.

\section{Liver Transplant Patient Imaging: Guidelines in the Pre-COVID-19 Era}

It is crucial to assess the transplant candidate timely by a multidisciplinary team, where the radiologist plays a key role in the early identification of anatomical conditions and disease before they could become symptomatic. The latest European guidelines on the management of the organ donor and the recipient [3] consider the following: 
(i) To acquire a precise evaluation of vascular anatomy

(ii) To acquire a precise evaluation of biliohepatic anatomy

(iii) To evaluate liver parenchymal status and dimensions

(iv) To reveal hidden cancer

(v) To exclude cholangiocarcinoma in primary sclerosing cholangitis patients

(vi) To evaluate posttransplantation complications

Pretransplant assessment is generally acquired by computed tomography (CT) imaging with intravenous contrast medium administration [3], but multiparametric ultrasound could provide a great contribution to recipient evaluation before and after surgery due to its noninvasiveness, widespread availability, high repeatability, and cost-effectiveness, alongside the option of a bedside execution.

2.1. Pretransplant Liver Imaging. An optimal multidisciplinary postoperative management by means of imaging modalities is recommended, with the three-phase intravenous contrast CT scan being the most adopted imaging method [3]. Additionally, transthoracic echocardiography in all LT candidates is valuable.

History of a treated cancer is not an absolute contraindication to LT. One of the eligibility criteria in cancer patients is the recurrence risk of less than $10 \%$ [3]. Another criterion is a free-of-recurrence interval time of 5 years, although it may vary considerably according to the type of malignancy.

Hepatocarcinoma (HCC) represents one of the main indications for transplantation by treating both the tumour and the underlying liver disease [4]. Not all HCCs can benefit from transplant treatment, but the effectiveness varies according to the stage of the disease, i.e., tumour stage, liver function, and functional status of the patient.

Both dynamic CT and dynamic magnetic resonance imaging (MRI) with contrast are the best imaging modalities to make HCC diagnosis, although they can underestimate or overestimate the extent in up to $25 \%$ of cases, compared with pathological findings of the explanted liver [5]. Extrahepatic staging should include chest CT and abdomen-pelvis CT or MRI.

While on the waiting list, periodic monitoring should be performed by imaging (dynamic CT, dynamic MRI, or contrast-enhanced ultrasound) and $\alpha$-fetoprotein measurements [4].

2.2. Posttransplant Liver Imaging. As a complex surgical procedure, accurate imaging is essential to detect early complications; therefore, regular diagnostic follow-up is recommended to improve graft and patient survival. LT complications could be classified into vascular (affecting the hepatic artery, portal vein, and/or hepatic vein) or biliary (involving the biliary tree). There is also a significant number of complications in relation to immunosuppression, more in details on infection, rejection, or malignancy [6].
While preoperative evaluation primarily involves CT and MRI, postoperative management relies on screening with US, eventually followed by deeper definition via additional modalities [7].

Within the first day postsurgery, US is highly recommended for baseline assessment $[8,9]$, representing the preferred investigation for an initial evaluation of symptomatic patients, especially in the case of young paediatric recipients, in order to obtain an early diagnosis of the postsurgical complication and carry out timely intervention [10].

Generally, in the postoperative period, a colour Doppler study is performed within 24 hours after an uncomplicated transplant, and another is required before discharge, but protocols may vary according to the different centres' practice.

A checklist of the findings to be evaluated could be followed during US examination to improve detection and to discern among the possible pathological conditions, as shown in Table 1 .

However, further evaluation may be necessary in case of doubt or inconclusive US examination (CT, MRI, or biopsy). Baheti et al. proposed a diagnostic algorithm for evaluating LT patients with suspected complications [6]. Also, Kimura et al. proposed a diagnostic algorithm to rapidly discover any vascular or biliary complications [9].

Oncological monitoring is relevant in LT patients; in fact, chronic immunosuppression could lead to the development of neoplastic diseases, such as posttransplant lymphoproliferative disorder (PTLD), Kaposi's sarcoma, and nonmelanocytic skin cancers. Observational studies have shown a 2-3-fold increased risk of solid organ cancers and a $>30$-fold increase in the rate of lymphoproliferative malignancies, compared to the general population [3].

PTLD related to Epstein-Barr virus infection consists of lymphoproliferative cancer, mainly occurring within the first 2 years of follow-up. In this case, abdominal involvement is more common than the extra-abdominal location, with singular or multiple lymphadenopathy manifestation and with or without splenomegaly. The most affected organs with extranodal involvement are the bowel, liver, kidneys, and adrenals, although it has been described as potentially occurring in any part of the human body [11, 12].

In transplant patients with hepatocellular carcinoma (HCC), recurrent HCC may also occur, usually involving the lung and the liver graft, with a median time to relapse of approximately 1 year [13].

Cancer screening must always be performed via endoscopy (colonoscopy, upper gastrointestinal endoscopy, and nasofibroscopy) and/or imaging (CT colonography, chest HRTC, and mammogram) tools, taking into consideration the patient's age, sex, alcohol consumption, and smoking habits.

For follow-up surveillance in patients with previous HCC, an international consensus report from 2012 recommends total-body CT or MRI every 6 to 12 months $[4,7]$.

Although there have been recent and significant improvements in US, even with contrast medium introduction (CEUS), US is less used than CT or MRI due to the inability to reliably acquire images of the entire liver during 
TABle 1: Posttransplant checklist based on US findings.

\begin{tabular}{|c|c|}
\hline \multirow{3}{*}{ Liver } & Size and volume \\
\hline & Focal lesions \\
\hline & $\begin{array}{l}\text { Hepatic echogenicity and } \\
\text { ecostructure }\end{array}$ \\
\hline \multirow{4}{*}{ Hepatic artery } & Anatomy \\
\hline & Patency and calibre \\
\hline & Parietal calcifications \\
\hline & $\begin{array}{l}\text { Doppler wave in inspiration } \\
\text { and after exhalation }\end{array}$ \\
\hline \multirow{6}{*}{ Portal system } & Anatomy \\
\hline & Patency and calibre \\
\hline & Doppler wave \\
\hline & Varices \\
\hline & Periportal oedema \\
\hline & Intraportal gas \\
\hline \multirow{3}{*}{ Biliary system } & Anatomy \\
\hline & Calibre \\
\hline & Pneumobilia \\
\hline $\begin{array}{l}\text { Vascular/biliary } \\
\text { anastomoses }\end{array}$ & $\begin{array}{l}\text { Details of vascular/ } \\
\text { biliary anastomoses }\end{array}$ \\
\hline \multirow{4}{*}{ Spleen } & Size and volume \\
\hline & Focal lesions \\
\hline & Splenic artery \\
\hline & Splenic vein \\
\hline \multirow{2}{*}{ Lymph nodes } & Periportal \\
\hline & Portacaval \\
\hline \multirow{2}{*}{ Other findings } & Ascites \\
\hline & Pleural effusion (right) \\
\hline
\end{tabular}

a particular contrast phase. However, there are many studies that demonstrate the usefulness of CEUS in the clinical and radiological management of the transplant patient, for example, in the kidney transplant scenario [14].

\section{New Ultrasound Techniques in Liver Transplantation}

3.1. Contrast-Enhanced Ultrasound. Colour Doppler US provides a reliable method for screening and evaluation of postliver transplant vasculature, but it has limitations in the assessment of small-calibre vessels. CEUS may overcome it, reducing the number of false-positive cases and thus unnecessary additional investigations, such as CT or angiography; in fact, by using microbubbles as blood pool contrast agents, CEUS allows qualitative and quantitative high-quality realtime evaluation.

CEUS can be used as a problem-solving tool when previous US, CT, and/or MRI imaging report undefined vascular and liver parenchymal findings [15]. In this regard, it could be very useful to visualize the hepatic artery, not otherwise recognizable in B-mode, for example, in conditions occur- ring with the overlapping of the portal vein or if of small calibre [16].

Another important benefit offered by CEUS is the detection of filling defects, if present, or lumen stenosis. As reported in the European Federation for Ultrasound in Medicine and Biology guidelines [17], CEUS is useful to identify portal thrombus hypervascularization which discriminates HCC portal invasion from bland portal vein thrombosis $[18,19]$.

Hepatic vein complications are rare (1\%) [20] and often difficult to be detected in US (such as in the case of hepatic vein stenosis); when the suspect arises, this requires confirmation via further imaging, generally in the form of CT or magnetic resonance imaging. CEUS might represent instead an exam demonstrating hepatic vein thrombosis or the site of anastomotic narrowing, without the need for further confirmation [16].

Concerning biliary complications, it has been previously reported that the administration of contrast media via the intravenous route is not of sufficient effectiveness, but its usefulness in demonstrating eventually biliary leakage and strictures increases when it is injected into the biliary system through a T-tube (CEUS cholangiography) [21, 22]. Finally, CEUS can also differentiate an infarcted area from an HCC lesion or abscess in the parenchyma.

For the abovementioned reasons, Como et al. suggest an extensive LT patient CEUS-integrated diagnostic workflow [15]. However, there are only few studies in the literature about CEUS applications in the LT setting, making it difficult to ascertain its own role or in combination with other traditional imaging methods, thus limiting significantly its use in the posttransplant setting [16, 23-27].

3.2. US Elastography. US elastography examines the intrinsic liver parenchymal state by measuring liver stiffness (LS) that, if high, is considered a marker of liver fibrosis or cirrhosis [28-30].

In the community, elastosonography is emerging as a noninvasive tool to evaluate the fibrosis of the hepatic graft, mainly in the paediatric population, allowing a decrease in the number of invasive procedures, such as liver biopsy.

US-based elastography comprehends different techniques $[28,29,31]$, with yet not an established specific role in LT patients. Current available data mainly report on the use of vibration-controlled transient elastography (TE) and point shear wave elastography (pSWE), especially regarding graft rejection.

The controlled attenuation parameter (CAP) is a novel ultrasound-based elastography method for detection of the grade of steatosis, an important parameter in the assessment of a liver donor, and mainly associated with male sex and high body mass index [32].

CAP measures ultrasound attenuation at the central frequency of the VCTE via the M and/or regular probe [33, 34]; its accuracy may be yet affected by variations in cut-off values of steatosis severity or integration with other parameters [35]; therefore, more recently and in other clinical settings, new quantification methods have been proposed. A recent meta-analysis looking at the TE and CAP role in 
steatosis and fibrosis diagnosis and staging in alcoholic and nonalcoholic liver diseases showed a pooled sensitivity and specificity of CAP of $0.84,0.83$, and 0.78 and $0.83,0.71$, and 0.62 for steatosis grades $\geq \mathrm{S} 1, \geq \mathrm{S} 2$, and $=\mathrm{S} 3$, respectively [36].

\section{Living Donor Imaging}

The living donor LT is less common in Western countries, in consideration of the potential risks for the donor and the higher posttransplant complication rate, in comparison to the deceased donation that is instead prevalent. It is yet very important and timely to emphasize the role of living donation to expand the donor pool and allow more transplants, especially in the postpandemic scenario, when due to current theatre restrictions, a growing organ demand is envisaged [37]. During COVID-19, in fact, it has been considered even a more essential resource for leading centres to contrast the mortality increase in candidates awaiting LT [38]. In the current global scenario, it is paramount to achieve an adequate imaging assessment, preferentially to be all performed in a single instance, avoiding in this way unnecessary donor exposure to risks related to hospital admission.

Generally, in standard practice, US is the first-line diagnostic tool to assess potential candidates and exclude focal hepatic lesions. It also assesses the amount of steatosis and provides preliminary vascular anatomy.

However, further assessment with cross-sectional imaging modalities is usually necessary.

CT findings determine split liver volumes, eventual vascular and biliary anatomy variations, and fatty infiltration [39-41]. In more detail, donor liver assessment includes [42] the following:

(i) Parenchymal evaluation for diffuse liver disease (fatty infiltration) and focal lesion detection

(ii) Liver volume estimation

(iii) Liver vascularization (arterial, portal, and hepatic venous) and variants

(iv) Biliary anatomy and variants

\section{Liver Transplantation in COVID-19: What the Pandemic Implied for Transplant Programs}

The suspension of the surgical activity, the burden of the infection in immunosuppressed patients, and the comorbidities underlying end-stage organ disease have impacted significantly the run of transplant programs. Uncertainty regarding SARS-CoV-2 transmission from the donor to the recipient and the risk of developing COVID-19 posttransplant from sources not related to the donor, as well as facility, logistical, and organizational issues, along with the implications of social distancing for the follow-up, represented the main issues to safely maintain an efficient transplant program [43]. Furthermore, the peak of COVID-19 hospitalization and admissions to intensive care units (ICUs) not only impacted the possibility to treat with dedicated pathways (buildings and staff) non-COVID-19 patients, such as transplant patients, but also importantly affected the overall number of deceased donors in ICU to be considered for the start of the whole transplantation process.

In parallel with the pandemic phases, we could identify three different approaches that have been adopted to carry on the necessary surgical activity [44]:

(1) Significant flattening of the observed pandemic curve allows a partial reopening. This includes a stable number of new cases and some stabilization in ward/ICU bed utilization (orange zone)

(2) The number of new COVID cases is flat or decreasing for a reasonable period, and ICU and ward bed utilization has been at a stable level at the transplant hospital (yellow zone)

(3) Prolonged stability and/or a decrease in COVID activity is observed along with prolonged stability or a decrease in hospital ICU/ward bed utilization. ICUs are running consistently below capacity in the transplant hospital (white zone)

In phases 1 and 2, liver transplant programs have limited living donor activity to moderate to severe sick recipients only and performed deceased donor activity only from standard donors or donors after cardiac death, with a higher chance of primary graft function (i.e., <50 years age). This occurred particularly at the beginning of the pandemic. Only in phase 3, living donor activity slowly came back to normal, with priority given to sicker recipients, while deceased donor activity was run under no restrictions. The distinction between living donation and deceased donation assumes that the former is at too high risk, since the prospective candidate is a healthy individual who does not need to come to the hospital, if not for the wish to donate. It is no surprise therefore that the elective phases (orange, yellow, and white zones) following the lockdown (red zone) impacted more substantially the kidney [45], a non-life-saving organ, in comparison to the liver. In more detail, in Italy, one of the most heavily affected countries by the pandemic, kidney waitlisted candidates dropped to $15 \%$ less in comparison to the constant number registered in the last 5 years; this effect was not instead observed for liver transplantation, with the numbers remaining stable throughout the pandemic, demonstrating the necessity to perform this life-saving procedure [46].

Indeed, it is paramount to correctly diagnose COVID-19 disease among transplant patients to prevent and manage this disease, with detrimental outcomes already described in the high-risk transplant subgroup.

It is known that underlying comorbidities increase the overall mortality risk in COVID-19 infection; therefore, patients with chronic liver disease, such as cirrhosis or autoimmune hepatitis, are more vulnerable. Yet, clinical risk factors specific for pathologic hepatic conditions and cancers and liver graft recipients are not clearly defined [47]. 
Mortality attributable to COVID-19 appears higher in patients with more advanced liver disease [47], and there is no reassuring data in terms of early immunization against the virus in transplanted patients [48]. While data is scarce, the incidence of early posttransplant Coronavirus Disease 2019 (COVID-19) may reach up to 38\% [49]; this is acquired generally in the community or during hospitalization. Thus, it was recommended that all patients should be evaluated for COVID-19 routinely and particularly preoperatively and that isolation should be considered for all transplant patients [50], with significant challenges to adequately follow them up.

5.1. Recipient and Donor SARS-CoV-2 Screening. A systematic SARS-CoV-2 screening strategy could be implemented for all recipients and donors including the following:

(i) A questionnaire on prehospitalization symptoms and a clinical examination at hospital admission

(ii) A nasopharyngeal swab for SARS-CoV-2 by RTPCR IP2/4

(iii) A chest CT scan prior to LT [51]

If all these three screening tests are negative, the LT could be performed.

Unfortunately, testing protocols are not standardized nationally, and current tests have significant false-negative results. Chest CT has a high sensitivity but is not always readily available, and society guidelines do not recommend its use as a routine screening strategy [52].

\subsection{Preoperative Imaging in Liver Transplantation during the COVID-19 Era}

5.2.1. Chest CT. Chest CT is a highly sensitive technique in identifying possible COVID-19 pulmonary involvement [53]. It is a widely used, fast-performing method that should be performed without contrast medium, unless pulmonary embolism is suspected. Its main limitations are the use of ionizing radiation and relatively high costs.

Many studies have shown that CT is more sensitive than the swab for the detection of SARS-CoV-2 infection, although it is not specific, since the same "ground glass" findings may be related to other interstitiopathies [54]. In this regard, a large Chinese analysis on 1014 patients reported lower sensitivity for RT-PCR, with a mean interval time between the initial negative result and the positive result of $5.1 \pm 1.5$ days, in contrast to the immediate positivity of the chest CT [55].

The European Society of Radiology (ESR) and the European Society of Thoracic Imaging (ESTI) published a document about SARS-CoV-2 pneumonia CT imaging focusing on typical findings in the early COVID-19 diagnosis [56].

According to ESR and ESTI guidelines [56], the most common COVID-19 pulmonary findings in unenhanced thin-section chest CT are as follows:

(i) Bilateral ground glass opacities, with a predominant peripheral, subpleural location (in the early phase), extensively located into the parenchyma or more focal, with a rounded shape

(ii) Intralobular reticulations superimposed on the ground glass opacities, resulting in a crazy-paving pattern (usually associated with a more severe stage of the disease)

Mucoid impactions, centrilobular nodules, lobar consolidation, lymphadenopathy, or significant pleural effusions are infrequently seen in COVID-19 patients.

The less common chest CT findings reported in $10 \%-70 \%$ of RT-PCR test-proven COVID-19 cases [57] are as follows: consolidation, linear opacity, septal thickening and/or reticulation, air bronchogram, pleural thickening, halo sign/reversed halo sign bronchiectasis, nodules, and bronchial wall thickening.

The distribution of these findings can be unilateral (15.0\%), multifocal (63.2\%), diffuse (26.4\%), and single and/ or focal $(10.5 \%)$, with a peripheral location only $(59.0 \%)$ or with a central and peripheral location concomitantly (36.2\%) [57]. The middle or upper lobe locations are more frequent.

The uncommon chest CT findings in RT-PCR testproven COVID-19 cases [57, 58] are as follows: pleural effusion, lymphadenopathy, tree-in-bud sign, central lesion distribution, pericardial effusion, and cavitating lung lesions.

Although the CT findings of COVID-19 are characteristic, the final diagnosis requires a positive RT-PCR test.

In the presence of $\mathrm{CT}$ changes highly suggestive of COVID-19, with an initial negative RT-PCR, it is important to repeat a second swab within the next 24-48 hours to exclude a false-negative result, particularly common at the early stages and before symptoms onset. The RT-PCR swab sensitivity depends on the type of test used, the throat swab quality, and the viral load. Patients with more severe infections typically have higher viral loads in all locations and shed the virus longer (21 versus 14 days) [56].

Whenever possible, chest CT should be done in dedicated rooms or at times with fewer exams to avoid other patients and staff exposure [59].

The normal chest CT result is estimated to be about $10.6 \%$ (95\% CI: 7.6\%, 13.7\%) in symptomatic COVID-19 patients [58]. Conversely, the normal chest CT incidence in asymptomatic COVID-19 patients is high and around $46 \%$ of patients [60].

In COVID-19 endemic areas, many studies suggest that an additional chest CT may be performed to help detect COVID-19 in patients who undergo extrathoracic CT [57]. These results can be observed in patients who underwent head and neck CT angiography [61-63], cervical or thoracic spine CT [61, 62], and abdomen CT [64-66]. At a later stage, a subsequent RT-PCR test to confirm COVID-19 diagnosis is required [67].

5.2.2. Chest Radiography. Chest radiography is not sensitive for COVID-19 pneumonia due to the low ability to detect ground glass opacities, which are the main COVID-19 imaging features. Its role, therefore, should be restricted to follow-up in ICU patients, who are too fragile to be 
sent for CT [56], particularly with the concomitant immunosuppression.

5.2.3. Chest Ultrasound. Several data indicate that lung US is a useful noninvasive bedside technique in the interstitial lung syndrome diagnosis and represents a reliable alternative to computed tomography (CT) during every step of COVID-19 disease, even before clinical manifestations [54, 68].

Unfortunately, chest US does not allow differentiation between bacterial pneumonia and viral pneumonia nor between pulmonary oedema and infection, and so its use should be limited to definitive-like cases only [69]. When US is unlikely to be diagnostic, CT should be preferred to minimize staff exposure. When instead US is recommended, for example, during pregnancy, in children or in ICU patients, it is a desirable bedside examination, reducing in this way the potential contamination of non-COVID areas during transport as well as the overall staff exposure [56].

Despite the wide availability, low cost, portability, and patient acceptability, particularly in those poorly cooperative, there are some limitations to an extensive US application in the setting of COVID-19, when compared to CT or X-ray [69]:

(1) Increased time and closer exposure for the operators to patients and vice versa, in comparison to other diagnostic modalities; therefore, US has the potential to increase the chances of infection

(2) Decontamination with liquid disinfectants is needed after COVID-19 examinations, and this might accumulate on the keyboard or in the command buttons

(3) Digital instrumentation, such as the transducers of the screen, could be potentially damaged using an aggressive decontamination liquid, and at the same time, the effectiveness of standard cleaning tissues could not be sufficient; the correct disinfection modalities are still unknown to some operators

(4) As for the case in the sterile theatre environment, the use of transparent, thin, disposable nylon bags might not entirely cover the US scanner but only the part in use at that given moment. Yet, this is not sufficient for the scanner to be utilized in non-COVID areas; thus, it is recommendable to provide each ward with a permanently located device, not to be shared with clean zones

(5) There is no evidence regarding the reproducibility and univocal interpretation of lung US in the assessment of SARS-CoV-2 pulmonary involvement, and we know interoperator reproducibility is a major concern when it comes to US. There might be a smooth learning curve, although this is only supposed since it is not ethical to recruit personnel to be exposed to COVID-19 only for testing purposes

5.3. Follow-Up Post-LT. Patients should be followed up in their own centres when necessary, although we strongly encourage avoiding hospital visits for routine control visits and recommend online consultation via telemedicine. Probably, this will continue also later on, in the postpandemic scenario, especially for patients with reasonable established maintenance, where they could feel more comfortable in avoiding in-person visits with healthcare professionals [70].

However, remote consultation is not sufficient for patients with evidence of posttransplant complications, who therefore should attend in-person clinic and standard visits with the transplant staff. Symptoms such as fever, cough, and dyspnoea could be an early alarm for COVID-19; therefore, screening in accordance with the national/local protocol is recommended to intervene as early as possible [71].

Finally, LT recipients and candidates should be considered a high priority for vaccination strategies: surgery per se is associated with worse outcomes $[2,72]$, and often the underlying comorbidities of end-stage liver disease patients represent additional risk factors for higher mortality.

\section{Conclusion}

LT relies on an appropriate imaging method for its success; during the COVID-19 pandemic, it has been demonstrated that early signs of SARS-CoV-2 infection could be only detected by means of chest CT and that other screening modalities are less accurate. The increasing impact of radiology to limit the virus spread, joint to the early management of the disease complications, reinforces the need for a multidisciplinary approach in LT candidates and recipients; these frail patients benefit from close radiological monitoring. In consideration also of the necessity to reduce in-person visits and follow-up, it is possible that new routine exams will be performed aiming to early capture post-LT complications. This will also be applied to LT candidates or living donors to limit their hospital access but in this way provide a comprehensive view of the medical conditions and surgical anatomy.

\section{Abbreviations}

CAP: $\quad$ Controlled attenuation parameter

COVID-19: Coronavirus Disease 2019

CT: $\quad$ Computed tomography

HCC: Hepatocarcinoma

ICU: Intensive care unit

LS: $\quad$ Liver stiffness

LT: $\quad$ Liver transplant

MRI: $\quad$ Magnetic resonance imaging

pSWE: $\quad$ Point shear wave elastography

RT-PCR: Reverse transcriptase-polymerase chain reaction

SARS-CoV-2: Severe Acute Respiratory Syndrome Coronavirus-type 2

SWE: $\quad$ Shear wave elastography

TE: $\quad$ Transient elastography

US: $\quad$ Ultrasound.

\section{Data Availability}

The data supporting this review have been provided throughout the text. 


\section{Conflicts of Interest}

The authors declare no conflict of interest with relevance to the present manuscript.

\section{References}

[1] G. A. Roth, S. Emmons-Bell, H. M. Alger et al., "Trends in patient characteristics and COVID-19 in-hospital mortality in the United States during the COVID-19 pandemic," JAMA Network Open, vol. 4, no. 5, pp. e218828-e218828, 2021.

[2] D. Nepogodiev, A. Bhangu, J. C. Glasbey et al., "Mortality and pulmonary complications in patients undergoing surgery with perioperative SARS-CoV-2 infection: an international cohort study," Lancet, vol. 396, no. 10243, pp. 27-38, 2020.

[3] EASL Clinical Practice Guidelines, "EASL clinical practice guidelines: liver transplantation," Journal of Hepatology, vol. 64, no. 2, pp. 433-485, 2016.

[4] P. A. Clavien, M. Lesurtel, P. M. Bossuyt et al., "Recommendations for liver transplantation for hepatocellular carcinoma: an international consensus conference report," The Lancet Oncology, vol. 13, no. 1, pp. e11-e22, 2012.

[5] N. Kneteman, T. Livraghi, D. Madoff, E. de Santibañez, and M. Kew, "Tools for monitoring patients with hepatocellular carcinoma on the waiting list and after liver transplantation," Liver Transplantation, vol. 17, Suppl 2, pp. S117-S127, 2011.

[6] A. D. Baheti, R. Sanyal, M. T. Heller, and P. Bhargava, "Surgical techniques and imaging complications of liver transplant," Radiologic Clinics of North America, vol. 54, no. 2, pp. 199215, 2016.

[7] M. M. Horrow, M. H. L. Huynh, M. M. Callaghan, and S. K. Rodgers, "Complications after liver transplant related to preexisting conditions: diagnosis, treatment, and prevention," Radiographics, vol. 40, no. 3, pp. 895-909, 2020.

[8] R. Sanyal, J. G. Zarzour, D. M. Ganeshan, P. Bhargava, C. G. Lall, and M. D. Little, "Postoperative Doppler evaluation of liver transplants," The Indian Journal of Radiology \& Imaging, vol. 24, no. 4, pp. 360-366, 2014.

[9] Y. Kimura, R. Tapia Sosa, D. Soto-Trujillo, Y. Kimura Sandoval, and C. Casian, "Liver transplant complications radiologist can't miss," Cureus, vol. 12, no. 6, article e8465, 2020.

[10] R. Girometti, G. Como, M. Bazzocchi, and C. Zuiani, "Postoperative imaging in liver transplantation: state-of-the-art and future perspectives," World Journal of Gastroenterology, vol. 20, no. 20, pp. 6180-6200, 2014.

[11] A. A. Borhani, K. Hosseinzadeh, O. Almusa, A. Furlan, and M. Nalesnik, "Imaging of posttransplantation lymphoproliferative disorder after solid organ transplantation," Radiographics, vol. 29, no. 4, pp. 981-1000, 2009.

[12] L. Wu, D. C. Rappaport, A. Hanbidge, N. Merchant, F. A. Shepherd, and P. D. Greig, "Lymphoproliferative disorders after liver transplantation: imaging features," Abdominal Imaging, vol. 26, no. 2, pp. 200-206, 2001.

[13] M. W. Welker, W. O. Bechstein, S. Zeuzem, and J. Trojan, "Recurrent hepatocellular carcinoma after liver transplantation - an emerging clinical challenge," Transplant International, vol. 26, no. 2, pp. 109-118, 2013.

[14] F. M. Drudi, F. Angelini, M. Bertolotto et al., "Role of contrastenhanced voiding urosonography in the evaluation of renal transplant reflux-comparison with voiding cystourethrography and a new classification," Ultraschall in der MedizinEuropean Journal of Ultrasound, 2021.
[15] G. Como, L. Montaldo, U. Baccarani, D. Lorenzin, C. Zuiani, and R. Girometti, "Contrast-enhanced ultrasound applications in liver transplant imaging," Abdominal Radiology, vol. 46, no. 1, pp. 84-95, 2021.

[16] Y. Goh, W. T. Neo, Y. M. Teo et al., "Role of contrastenhanced ultrasound in the evaluation of post-liver transplant vasculature," Clinical Radiology, vol. 75, no. 11, pp. 832-844, 2020.

[17] C. F. Dietrich, C. P. Nolsøe, R. G. Barr et al., "Guidelines and good clinical practice recommendations for contrast enhanced ultrasound (CEUS) in the liver - update 2020 - WFUMB in cooperation with EFSUMB, AFSUMB, AIUM, and FLAUS," Ultraschall in der Medizin-European Journal of Ultrasound, vol. 41, no. 5, pp. 562-585, 2020.

[18] P. R. Galle, A. Forner, J. M. Llovet et al., "EASL clinical practice guidelines: management of hepatocellular carcinoma," Journal of Hepatology, vol. 69, no. 1, pp. 182-236, 2018.

[19] L. Tarantino, P. Ambrosino, and M. N. Di Minno, "Contrastenhanced ultrasound in differentiating malignant from benign portal vein thrombosis in hepatocellular carcinoma," World Journal of Gastroenterology, vol. 21, no. 32, pp. 9457-9460, 2015.

[20] P. Wozney, A. B. Zajko, K. M. Bron, S. Point, and T. E. Starzl, "Vascular complications after liver transplantation: a 5-year experience," AJR. American Journal of Roentgenology, vol. 147, no. 4, pp. 657-663, 1986.

[21] S. S. Chopra, R. Eisele, L. Stelter et al., "Contrast enhanced ultrasound cholangiography via T-tube following liver transplantation," Annals of Transplantation, vol. 17, no. 4, pp. 108-112, 2012.

[22] J. Ren, B. W. Zheng, P. Wang et al., "Revealing impaired blood supply to the bile ducts on contrast-enhanced ultrasound: a novel diagnosis method to ischemic-type biliary lesions after orthotropic liver transplantation," Ultrasound in Medicine \& Biology, vol. 39, no. 5, pp. 753-760, 2013.

[23] P. S. Sidhu, A. S. Shaw, J. B. Karani, S. M. Ryan, and S. M. Ellis, "Microbubble ultrasound contrast in the assessment of hepatic artery patency following liver transplantation: role in reducing frequency of hepatic artery arteriography," European Radiology, vol. 14, no. 1, pp. 21-30, 2004.

[24] A. E. Berstad, K. Brabrand, and A. Foss, "Clinical utility of microbubble contrast-enhanced ultrasound in the diagnosis of hepatic artery occlusion after liver transplantation," Transplant International, vol. 22, no. 10, pp. 954-960, 2009.

[25] S. J. Lee, K. W. Kim, S. Y. Kim et al., "Contrast-enhanced sonography for screening of vascular complication in recipients following living donor liver transplantation," Journal of Clinical Ultrasound, vol. 41, no. 5, pp. 305-312, 2013.

[26] J. Rennert, C. Dornia, M. Georgieva et al., "Identification of early complications following liver transplantation using contrast enhanced ultrasound (CEUS). First results," Journal of Gastrointestinal \& Liver Diseases, vol. 21, no. 4, pp. 407-412, 2012.

[27] R. Q. Zheng, R. Mao, J. Ren et al., "Contrast-enhanced ultrasound for the evaluation of hepatic artery stenosis after liver transplantation: potential role in changing the clinical algorithm," Liver Transplantation, vol. 16, no. 6, pp. 729-735, 2010.

[28] C. F. Dietrich, J. Bamber, A. Berzigotti et al., "EFSUMB guidelines and recommendations on the clinical use of liver ultrasound elastography, update 2017 (long version)," Ultraschall 
in der Medizin-European Journal of Ultrasound, vol. 38, no. 4, pp. e16-e47, 2017.

[29] L. Mulazzani, V. Cantisani, and F. Piscaglia, "Different techniques for ultrasound liver elastography," Journal of Hepatology, vol. 70, no. 3, pp. 545-547, 2019.

[30] F. Piscaglia, V. Salvatore, L. Mulazzani et al., "Differences in liver stiffness values obtained with new ultrasound elastography machines and FibroScan: a comparative study," Digestive and Liver Disease, vol. 49, no. 7, pp. 802-808, 2017.

[31] J. Bamber, D. Cosgrove, C. F. Dietrich et al., "EFSUMB guidelines and recommendations on the clinical use of ultrasound elastography. Part 1: basic principles and technology," Ultraschall in der Medizin-European Journal of Ultrasound, vol. 34, no. 2, pp. 169-184, 2013.

[32] W. Fang, M. Noda, K. Gotoh et al., "Fatty liver disease in living liver donors: a single-institute experience of 220 donors," Transplant International, vol. 34, no. 11, pp. 2238-2246, 2021.

[33] M. Sasso, M. Beaugrand, V. de Ledinghen et al., "Controlled attenuation parameter (CAP): a novel $\mathrm{VCTE}^{\mathrm{TM}}$ guided ultrasonic attenuation measurement for the evaluation of hepatic steatosis: preliminary study and validation in a cohort of patients with chronic liver disease from various causes," Ultrasound in Medicine \& Biology, vol. 36, no. 11, pp. 1825-1835, 2010.

[34] M. Sasso, V. Miette, L. Sandrin, and M. Beaugrand, "The controlled attenuation parameter (CAP): a novel tool for the non-invasive evaluation of steatosis using FibroScan ", Clinics and Research in Hepatology and Gastroenterology, vol. 36, no. 1, pp. 13-20, 2012.

[35] V. de Lédinghen, J. Vergniol, M. Capdepont et al., "Controlled attenuation parameter (CAP) for the diagnosis of steatosis: a prospective study of 5323 examinations," Journal of Hepatology, vol. 60, no. 5, pp. 1026-1031, 2014.

[36] C. Cai, X. Song, X. Chen et al., "Transient elastography in alcoholic liver disease and nonalcoholic fatty liver disease: a systemic review and meta-analysis," Canadian Journal of Gastroenterology \& Hepatology, vol. 2021, article 8859338, pp. 1-10, 2021.

[37] C. Quintini, K. Hashimoto, T. D. Uso, and C. Miller, "Is there an advantage of living over deceased donation in liver transplantation?," Transplant International, vol. 26, no. 1, pp. 1119, 2013.

[38] S. di Sandro, P. Magistri, V. Bagnardi, B. Catellani, G. P. Guerrini, and F. di Benedetto, "The COVID-19 second wave risk and liver transplantation: lesson from the recent past and the unavoidable need of living donors," Transplant International, vol. 34, no. 3, pp. 585-587, 2021.

[39] H. P. Grewal, M. H. Shokouh-Amiri, S. Vera, R. Stratta, W. Bagous, and A. O. Gaber, "Surgical technique for right lobe adult living donor liver transplantation without venovenous bypass or portocaval shunting and with duct-to-duct biliary reconstruction," Annals of Surgery, vol. 233, no. 4, pp. 502508, 2001.

[40] R. S. Brown Jr., "Live donors in liver transplantation," Gastroenterology, vol. 134, no. 6, pp. 1802-1813, 2008.

[41] S. Nadalin, M. Bockhorn, M. Malagó, C. Valentin-Gamazo, A. Frilling, and C. E. Broelsch, "Living donor liver transplantation," HPB: The Official Journal of the International Hepato Pancreato Biliary Association, vol. 8, no. 1, pp. 10-21, 2006.

[42] D. Sahani, R. D'souza, R. Kadavigere et al., "Evaluation of living liver transplant donors: method for precise anatomic defi- nition by using a dedicated contrast-enhanced MR imaging protocol," Radiographics, vol. 24, no. 4, pp. 957-967, 2004.

[43] M. I. Bellini, C. Lewis, N. Welch, I. Anderson, and V. Papalois, "COVID-19 and surgical life: cross-sectional survey," British Journal of Surgery, vol. 108, no. 4, pp. e177-e178, 2021.

[44] Contributors to the $\mathrm{C} 4$ article, "C4 article: Implications of COVID-19 in transplantation," American Journal of Transplantation, vol. 21, no. 5, pp. 1801-1815, 2021.

[45] M. I. Bellini, F. Tortorici, and M. Capogni, "Kidney transplantation and the lock-down effect," Transplant International, vol. 33, no. 9, pp. 1142-1143, 2020.

[46] M. I. Bellini, F. Tortorici, and M. Capogni, "COVID-19 in solid organ transplantation: an analysis of the impact on transplant activity and wait lists," Transplant International, vol. 34, no. 1, pp. 209-212, 2021.

[47] O. K. Fix, B. Hameed, R. J. Fontana et al., "Clinical best practice advice for hepatology and liver transplant providers during the COVID-19 pandemic: AASLD expert panel consensus statement," Hepatology, vol. 72, no. 1, pp. 287-304, 2020.

[48] B. J. Boyarsky, T. Po-Yu Chiang, W. A. Werbel et al., "Early impact of COVID-19 on transplant center practices and policies in the United States," American Journal of Transplantation, vol. 20, no. 7, pp. 1809-1818, 2020.

[49] E. Abdala, D. R. Waisberg, L. B. Haddad et al., "Enabling liver transplantation during the COVID-19 era: more than screening donors and recipients for SARS-CoV-2," Clinics (São Paulo, Brazil), vol. 75, p. e2377, 2020.

[50] P. Eslami, M. Moradi, A. Dooghaie Moghadam et al., "Lethal outcome of COVID-19 pneumonia in a new liver recipient with neurological manifestation," Gastroenterology and Hepatology From Bed to Bench, vol. 13, no. 4, pp. 405-409, 2020.

[51] X. Muller, G. Tilmans, Q. Chenevas-Paule et al., "Strategies for liver transplantation during the SARS-CoV-2 outbreak: preliminary experience from a single center in France," American Journal of Transplantation, vol. 20, no. 11, pp. 2989-2996, 2020.

[52] American College of Radiology, "ACR recommendations for the use of chest radiography and computed tomography (CT) for suspected COVID-19 infection 2020," March 2020, https:// www.acr.org/Advocacy-and-Economics/ACR-PositionStatements/Recommendations-for-Chest-Radiography-andCT-for-Suspected-COVID19-Infection.

[53] M.-Y. Ng, E. Y. Lee, J. Yang et al., "Imaging profile of the COVID-19 infection: radiologic findings and literature review," Radiology: Cardiothoracic Imaging, vol. 2, no. 1, article e200034, 2020.

[54] Y. Li and L. Xia, "Coronavirus disease 2019 (COVID-19): role of chest CT in diagnosis and management," AJR. American Journal of Roentgenology, vol. 214, no. 6, pp. 1280-1286, 2020.

[55] T. Ai, Z. Yang, H. Hou et al., "Correlation of chest CT and RTPCR testing for coronavirus disease 2019 (COVID-19) in China: a report of 1014 cases," Radiology, vol. 296, no. 2, pp. E32-e40, 2020.

[56] on behalf of the European Society of Radiology (ESR) and the European Society of Thoracic Imaging (ESTI), M. P. Revel, A. P. Parkar et al., "COVID-19 patients and the radiology department - advice from the European Society of Radiology (ESR) and the European Society of Thoracic Imaging (ESTI)," European Radiology, vol. 30, no. 9, pp. 4903-4909, 2020. 
[57] T. C. Kwee and R. M. Kwee, "Chest CT in COVID-19: what the radiologist needs to know," Radiographics, vol. 40, no. 7 , pp. 1848-1865, 2020.

[58] H. J. A. Adams, T. C. Kwee, D. Yakar, M. D. Hope, and R. M. Kwee, "Chest CT imaging signature of coronavirus disease 2019 infection: in pursuit of the scientific evidence," Chest, vol. 158 , no. 5 , pp. 1885-1895, 2020.

[59] M. Mossa-Basha, J. Medverd, K. F. Linnau et al., "Policies and guidelines for COVID-19 preparedness: experiences from the University of Washington," Radiology, vol. 296, no. 2, pp. E26-e31, 2020.

[60] S. Inui, A. Fujikawa, M. Jitsu et al., "Chest CT findings in cases from the cruise ship Diamond Princess with coronavirus disease (COVID-19)," Imaging, vol. 2, no. 2, article e200110, 2020.

[61] R. Jain, M. Young, S. Dogra, H. Kennedy, V. Nguyen, and E. Raz, "Surprise diagnosis of COVID-19 following neuroimaging evaluation for unrelated reasons during the pandemic in hot spots," AJNR. American Journal of Neuroradiology, vol. 41, no. 7, pp. 1177-1178, 2020.

[62] R. M. Kwee, J. Krdzalic, B. A. C. M. Fasen, T. M. H. de Jaegere, and for the COVID-19 CT Investigators South-East Netherlands (CISEN) Study Group, "CT scanning in suspected stroke or head trauma: is it worth going the extra mile and including the chest to screen for COVID-19 infection?," AJNR. American Journal of Neuroradiology, vol. 41, no. 7, pp. 1165-1169, 2020.

[63] S. Kihira, J. Schefflein, M. Chung et al., "Incidental COVID-19 related lung apical findings on stroke CTA during the COVID-19 pandemic," Journal of Neurointerventional Surgery, vol. 12, no. 7, pp. 669-672, 2020.

[64] R. Hossain, M. S. Lazarus, A. Roudenko et al., "CT scans obtained for nonpulmonary indications: associated respiratory findings of COVID-19," Radiology, vol. 296, no. 3, pp. E173e179, 2020.

[65] B. Dane, G. Brusca-Augello, D. Kim, and D. S. Katz, "Unexpected findings of coronavirus disease (COVID-19) at the lung bases on abdominopelvic CT," AJR. American Journal of Roentgenology, vol. 215, no. 3, pp. 603-606, 2020.

[66] A. Siegel, P. J. Chang, Z. J. Jarou et al., "Lung base findings of coronavirus disease (COVID-19) on abdominal CT in patients with predominant gastrointestinal symptoms," AJR. American Journal of Roentgenology, vol. 215, no. 3, pp. 607-609, 2020.

[67] G. D. Rubin, C. J. Ryerson, L. B. Haramati et al., "The role of chest imaging in patient management during the COVID-19 pandemic: a multinational consensus statement from the Fleischner Society," Chest, vol. 158, no. 1, pp. 106-116, 2020.

[68] M. Allinovi, A. Parise, M. Giacalone et al., "Lung ultrasound may support diagnosis and monitoring of COVID-19 pneumonia," Ultrasound in Medicine \& Biology, vol. 46, no. 11, pp. 2908-2917, 2020.

[69] F. Piscaglia, F. Stefanini, V. Cantisani et al., "Benefits, open questions and challenges of the use of ultrasound in the COVID-19 pandemic era. The views of a panel of worldwide international experts," Ultraschall in der Medizin-European Journal of Ultrasound, vol. 41, no. 3, pp. 228-236, 2020.

[70] M. I. Bellini, L. Pengel, L. Potena, L. Segantini, and ESOT COVID-19 Working Group, "COVID-19 and education: restructuring after the pandemic," Transplant International, vol. 34, no. 2, pp. 220-223, 2021.
[71] S. Saigal, S. Gupta, S. Sudhindran et al., "Liver transplantation and COVID-19 (coronavirus) infection: guidelines of the Liver Transplant Society of India (LTSI)," Hepatology International, vol. 14, no. 4, pp. 429-431, 2020.

[72] COVIDSurg Collaborative and GlobalSurg Collaborative, "SARS-CoV-2 vaccination modelling for safe surgery to save lives: data from an international prospective cohort study," British Journal of Surgery, vol. 108, no. 9, pp. 1056-1063, 2021. 